\title{
Analysis and Discussion about Wireless LAN Technology
}

\author{
Zhongtang $\mathrm{Li}^{1}$, Yin Lei ${ }^{2}$ \\ ${ }^{1}$ Jiangxi College of Applied Technology, Gan Zhou, Jiang Xi, 341000 \\ ${ }^{2}$ Gannan Medical University, Gan Zhou, Jiang Xi, 341000
}

KEYWORDS: Wireless LAN Technology, Analysis, Discussion

\begin{abstract}
Effect of wireless LAN technology to China mobile and portable communication is enormous, mainly using wireless LAN network unlimited channel signal transmission. WLAN has information transmission convenient network of flexible benefits. This paper discusses the recent rapid development of wireless LAN technology. By contrast the wireless LAN with the wired network, wireless LAN technology standards, wireless LAN topology, analyzes the principles and techniques used in wireless LAN. Provide technical support for the selection of the formation of the wireless LAN.
\end{abstract}

\section{Introduction}

After entering the 1990s, with the widespread use of powerful, portable computer, there is an urgent need for it to carry a portable machine is still able to maintain their access to the office local area network in place outside the office, or access to other public networks. Especially with the development of Bluetooth without surgery and related technology, the range of the wireless LAN increases, has developed into a new stage of the mobile computer network. Wireless LAN is a computer network and wireless communication technology product of the combination. From a professional point of view, the use of wireless local area network is an effective method for wireless multiple-access channel to support communication between computers, and provides the possibility for mobile, personalized communications and multimedia applications.

Communication between computers and networks are combined to form a wireless LAN technology, application of the technology, so that users are able to realize that no matter at any time data communication. Wireless LAN biggest advantage is not limited by the kind of cable network cabling. Now the country has been largely achieved wireless LAN coverage, in this case people have experienced the wireless LAN to work and life convenience. WLAN broad range of applications, not only allows users to establish long-distance voice and data connections, you can also set up close radio frequency technology. Therefore, the application of wireless network technology enables flexibility and scalability of the network increases.

\section{The Concept of Wireless LAN}

Wireless LAN is the use of infrared technology consisting of radio frequency wireless local area network, which replaces the traditional coaxial cable networks. Based wireless network is wireless networking capabilities via modem, AP, wireless bridges, hubs, as well as wireless LAN, wireless LAN comes with a wireless signal transmitting and receiving functions, to facilitate the sharing of resources between computers, you can achieve network support all services. 
Wireless LAN cabling and wiring will not be restricted areas, mobility is very strong in terms of data transfer rate of more than $1 \mathrm{Mbit} / \mathrm{s}$, the transmission rate is very fast, and very low error rate transmission. After the transfer is complete, users can extract data to ensure data is not compromised. Wireless LAN can be combined into a variety of network topologies, easy installation requirements of various applications. Wireless LANs based on the IEEE802.11 protocol, fully compatible with the network of mainstream NOS. Much network software can run without modification on the wireless LAN. Wireless LAN network maintenance and repair time is not required to check the wiring, saving the use of funds.

\section{Features of Wireless Local Area Network}

Compared with wired networks, wireless local area network has many advantages, mainly in the following aspects.

In the network construction, the longest construction period, the greatest impact on the surrounding environment, that is, network cabling construction projects. During the construction process, often requires digging, pipe threading frame. The biggest advantage of WLAN eliminates or reduces the workload of network cabling, generally as long as the installation of one or more access points devices, local area network can cover the entire building or area.

Due to lack of flexibility in a wired network, which requires the network planners to consider possible future development needs, which often leads to a large number of preset information points lower utilization. Once the network developed beyond the design and planning, but also to spend more costs for network transformation, and the WLAN to avoid or reduce the occurrence of the above circumstances. In addition, the use of wireless LAN to avoid the high cost of cable installation fees, monthly charges of leased lines and equipment need to be moved when the increase in related costs.

Workers EEE802.n from wireless network standards, we can know that the wireless network supports 2.4GH: band next 13 sub channels, each channel occupies a bandwidth of $22 \mathrm{MHz}$, and can also have three completely exclusive sub-channel in the $2.4 \mathrm{GHz}$ frequency band, and in within each sub channel, based on n-bit random symbols to each base station sub-coding user access to multi-user channel reuse. In this mode, some wireless networks can allow up to 80 users to access. The cable network will suffer HUB number of interfaces, and the wiring length and so many limits.

WLAN has a variety of configurations, the flexibility to choose according to need. Thus, WLAN can handle only a few users from small LAN to a large network of thousands of users, and can provide characteristics like "roaming (Roaming)" and other cable networks can not provide. Reliable communication, strong anti-interference performance, with the ideal receiver sensitivity, antenna can provide a wide range of powerful and reliable wireless transmission.

Wireless LAN settings allow users at any time, anywhere access to network data, no need to specify explicit access sites, so users can roam in the network. A limiting high mobility, communication range from environmental conditions, broadens the scope of the transmission network.

\section{Comparison between Wireless LAN and Wired LAN}

Wireless LAN is an extension of limited LAN, but can not replace wired LANs, we compare the two from the following point of view what is the difference:

From the condition of building a network point of view, compared to the wired LAN wireless LAN, set up, configure, and easier maintenance, general computer staff competent management of 
the network. Wireless LAN and constraints from the environment, can not be wiring or wiring where networking is more difficult, for example, contain hazardous materials plant, offshore fleet and the like.

But in fact, on the one hand as the technology matures, the price of wireless networking devices drop, on the other hand can be omitted, wiring wireless networking problems, especially in some of the cost of wiring is relatively large (such as an offshore submarine cabling networking needs), wireless LAN saves wiring costs. Therefore, from a comprehensive perspective, the average cost of wireless networking is even lower than the wired network.

From the performance point of view, it is clear that wireless LAN performance is relatively low, the current wireless LAN bandwidth is limited by factors such as limited, slow, kind of individual LANs (for example, Infrared company) may have a greater throughput reached the wired LAN speed. Wireless LAN speed data radio frequencies with improved technology will inevitably increase, while in the study of the higher microwave frequencies transmitted. In addition, wireless local area network typically slower reasons but also because the wireless communication over a wired link more susceptible to interference and some, it is more error prone. Therefore, the need to increase wireless data transmission error correction checksum. This resulted in overhead communication process, increasing the bandwidth reduces the effective throughput. Therefore, wireless LAN and wired LAN both have their own advantages.

\section{Standard Protocol for Wireless Local Area Network}

IEEE802.11 is a landmark protocol standard and the protocol standards can not only make a variety of wireless products for Internet, you can also use a single chip to solve the various problems of the entire wireless LAN cost reduced. IEEE802.11 protocol standard accepted by many in the environment, all manufacturers of wireless LAN products based on this protocol has the basic product, but the transmission rate IEEE802.11 protocol is limited, can only reach $2 \mathrm{Mbit} / \mathrm{s}$, the transfer rate is slower, so on IEEE research group has developed a a and b are two standards, the highest transmission speed IEEE802.11b newly developed can reach 11Mbit / s, a standard or even willing to reach 54Mbit / s, fully meet the needs of any network.

IrDA protocol standards are point to point communications using infrared technology to transmit data, the power of this technology is relatively low, is still relatively small device applications, supported by mobile communications, in terms of hardware and software technologies are very mature. IrDA protocol standard network transmission speeds of up to 16Mbit / s, IrDA protocol interface is now essential that all laptops are installed, but now the market has launched IrDA device is connected via USB to the computer. IrDA protocol standards but only for two devices that have ports for data transmission, and transmission in the middle of the two devices can not have any obstacles, so this is the biggest flaw of the IrDA protocol and IrDA infrared radiation is not life particularly long, if long-term use might be scrapped.

Home RF protocol standard for home networking and design, combined with the IEEE802.11 protocol, reduces the cost of call data between home users, Home RF operating frequency band is mainly in the vicinity of $2.4 \mathrm{GHz}$, allowing four voice channels to achieve high-quality calls, but Home RF network data transfer rate can only reach 2Mbit / s, need to be improved.

\section{Application of Wireless LAN Technology}

Warehouse stocking and delivery of goods in the retail industry can use wireless LAN technology, barcode all goods will be reading, LAN to a computer inside the warehouse to connect, easy to use wireless LANs goods inventory, and updated in real time to store data and network data list. 
Wireless LAN applications in the transport industry is also very wide, many of the transport industry can not lay a large cargo crane cable channel, it will use a walkie-talkie and man-made transport cargo and article, this approach is prone to error cargo transport . Therefore, the wireless LAN is widely used wireless LAN can carry cargo and computer input goods number, and updated in real time by computer processing, improve efficiency to ensure the quality of work.

Changes in the financial information industry very quickly, users can take advantage of all the financial transfer and sale of hand-held communication devices connected to a wireless LAN network data changes to financial products; of personnel can also use mobile communication devices to conduct financial product transactions.

Application of wireless local area network in the office environment can improve office efficiency, office staff can query information anytime, anywhere via the wireless LAN, and each office staff can use wireless LAN for data transmission, make the office more convenient.

\section{Conclusion}

At present, the coverage of the wireless LAN is growing, more and more high transmission rate, the wireless LAN has become a broadband network solutions, and application of the technology will be around the world, achieve worldwide undifferentiated cover

\section{Reference}

[1] Wu Zongzhe. Application of Wireless Network Technology in Ship Navigation System [J] Harbin: Harbin Engineering University, 2013.

[2] Ran Run concentration based wireless LAN technology for mobile medical system design and implementation Now [J] Xiamen: Xiamen University, 2013.

[3] Lou Xiaoqian research. WLAN MAC layer protocol technology and back off [J] Shenyang: Northeastern University, 2012.

[4] China wireless LAN technology in ship information construction of [J]. Qingdao Ocean Shipping Mariners College, 2009 (2): 1-4,40.

[5] Wang Jian Application of Wireless LAN technology in wind farms in the [J]. Chengdu: University of Electronic Science and Technology, 2010.

[6] Leibo wireless LAN technology development status and future trends in research [J]. Power Device Technology and Software Engineering, 2014 (6): 41. 